\title{
Development of a Light Detector for a Low Temperature Scanning Tunneling Microscope
}

\author{
Luiza Lober de Souza Piva*, Yves Maia Auad, Luiz F. Zagonel
}

\begin{abstract}
This project aims to implement, test and improve a light detection system that will be installed in a Scanning Tunneling Microscope operating at Low Temperatures (LT-STM). This system is made of a parabolic mirror, a converging lens and an optical fiber bundle. The challenge will be to aligning these elements with sub-micrometric precision and develop alignment protocols to align the mirror with respect to the tunnel junction. Such procedures are crucial to the Young Investigators Project that funds the purchase of the LT-STM and which the final objective is the study of the luminescence of nanostructured semiconductors.
\end{abstract}

\section{Key words:}

Optics, Instrumentation, LT-STM

\section{Introduction}

Though surface scanning tecniques, it is possible to explore the potential of a given semiconductor surface to generate different types of luminescences. In this project, the focus will be given to cathodoluminescence light emission.

An optical system will then be developed to collect those luminescent signals with high precision, given that the measurements made by the STM will be highly dependent with the collection's efficiency. The approach to this challenge is to use a highly concave parabolical mirror as the signal collector, with $72 \%$ efficiency in light collection as shown by computational simulations carried by the research group - the biggest when compared to other options studied before implementation ${ }^{1}$. The system will also be composed of convergent lenses, optical fibers and a spectrometer for signal analysis.

The greatest challenge of this project will be to adequate the system to the optical and termomechanical restrictions imposed by the STM in a UHV environment.

\section{Results and Discussion}

Using optical theory ${ }^{2}$ to define the criteria of alignment of the components of the optical detector, as schematized below in Img.1, it was possible to assemble the system such that the mirror could be displaced with nanometric precision $^{3}$ in all three axis, with a piezoelectric manipulator specifically developed to that end; it's also possible to reposition the aspherical lens so that both components are aligned. The later will be responsible to correct the aspherical aberration expected from the highly concave mirror. A optical fiber will then be assembled jointly with the lens.

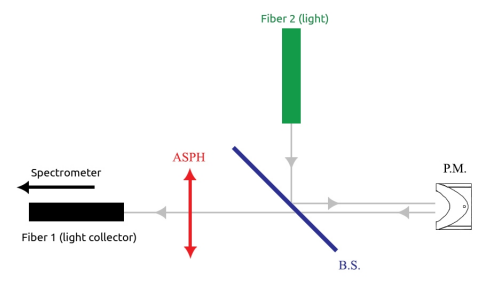

Image 1. Schematic drawing of the assembly of key components to the optical system.

Initially, the alignment will be verified using CMOS cameras instead of a fiber. These will allow the analysis of the signal's intensity though a matrix of pixels, resulting in a histogram. With multiple images of varying mirror's position and a steady signal, it's then possible to construct a graph such as Img. 2, correlating maximum pixel counts vs relative mirror's position, defining then the full width at half maximum of such measurements. This results in the maximum precision of alignment at a given axis.

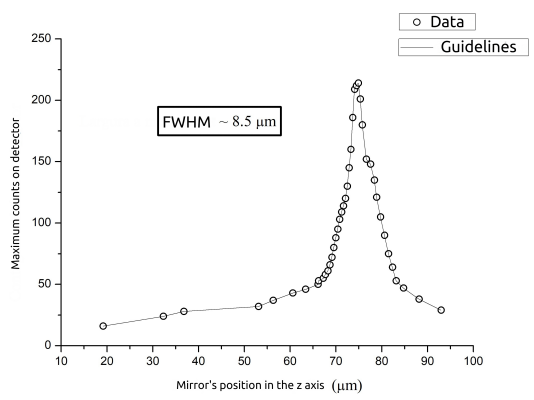

Image 2. FWHM relative to the $z$ (vertical) axis

Given that the estimated value to this measurement was of $5 \mu \mathrm{m}$ and, as shown above, a FWHM of $8,5 \mu \mathrm{m}$ was obtained though the described methodology, we can ensure that the luminescent spot of light can be known with relative accuracy.

\section{Conclusions}

The protocol developed by this project showed to be a interesting approach to predict the behavior of light propagating in free space and restrained to the optical system proposed. At the moment, the system is being installed and readied to be used in the LT-STM. The data collected after that will then be presented and compared with the results previously obtained.

\section{Acknowledgement}

This work was possible due to FAPESP's sponsorship to this project, which is associated to a Young Investigators Project sponsored by the same agency.

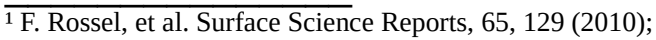

${ }^{2}$ Saleh, B. and Teich, M. (2013). Fundamentals of photonics. Chicester: Wiley

${ }^{3}$ W.M. Kuo, S.F. Chuang, C.Y. Nian, Y.S. Tarng (2008). Precision nanoalignment system using machine vision with motion controlled by piezoelectric motor. Mechatronics, Vol. 18, págs. 21-34. ISSN 0957-4158 